Supplement of Biogeosciences Discuss., 11, 12937-12983, 2014

http://www.biogeosciences-discuss.net/11/12937/2014/

doi:10.5194/bgd-11-12937-2014-supplement

(C) Author(s) 2014. CC Attribution 3.0 License.

(c) (i)

Supplement of

\title{
Carbon dioxide flux and net primary production of a boreal treed bog: responses to warming and water table manipulations
}

T. M. Munir et al.

Correspondence to: T. M. Munir (tmmunir@ucalgary.ca) 
Supplement Table S1: Estimated parameter values ( \pm SE), standard error of the estimate (SEE) and goodness of fit $\left(r^{2}\right)$ for the gross primary production of forest Floor $\left(\mathrm{GPP}_{\mathrm{ff}}\right)$ model (Eq. 1) ${ }^{\mathrm{a}}$.

\begin{tabular}{|c|c|c|c|c|c|c|c|c|c|}
\hline \multirow[t]{2}{*}{ Yr } & \multirow[t]{2}{*}{ Site / Microform } & \multicolumn{2}{|c|}{ GPP $_{\text {ff }}$ vs. PAR } & \multicolumn{2}{|c|}{ GPP $_{\text {ff }}$ vs. WT } & \multicolumn{2}{|c|}{ GPP $_{\mathrm{ff}}$ Vs. $T$} & \multirow{2}{*}{$\begin{array}{c}\text { SEE } \\
\mathrm{g} \mathrm{CO}_{2} \mathrm{~m}^{-2} \mathrm{~d}^{-1}\end{array}$} & \multirow[t]{2}{*}{$r^{2}$} \\
\hline & & $\begin{array}{c}P_{\max } \\
\text { g CO}_{2} \mathrm{~m}^{-2} \mathbf{d}^{-1}\end{array}$ & $\underset{\mu \mathrm{mol} \mathrm{m}^{-2} \mathrm{~s}^{-1}}{k}$ & $\begin{array}{c}\mathbf{W T}_{\text {opt }} \\
\mathbf{c m}\end{array}$ & $\begin{array}{l}\mathbf{W T}_{\text {tol }} \\
\mathbf{c m}\end{array}$ & ${ }^{T_{\text {opt }}}$ & $\begin{array}{l}T_{\text {tol }} \\
{ }^{\circ} \mathrm{C}\end{array}$ & & \\
\hline \multirow[t]{15}{*}{2011} & CONTROL & & & & & & & & \\
\hline & Ambient Hummock & $-22.2 \pm 4$ & $900 \pm 422$ & $-56 \pm 13$ & $30 \pm 16$ & $16 \pm 4$ & $10 \pm 5$ & 2.3 & 0.83 \\
\hline & Warmed Hummock & $-27.2 \pm 6$ & $999 \pm 468$ & $-48 \pm 4$ & $15 \pm 11$ & $9 \pm 15$ & $20 \pm 16$ & 3.4 & 0.77 \\
\hline & Ambient Hollow & $-19.4 \pm 7$ & $950 \pm 601$ & $-28 \pm 10$ & $23 \pm 21$ & $14 \pm 8$ & $10 \pm 13$ & 2.0 & 0.63 \\
\hline & Warmed Hollow & $-15.7 \pm 4$ & $500 \pm 318$ & $-36 \pm 2$ & $11 \pm 3$ & $17 \pm 13$ & $18 \pm 30$ & 3.0 & 0.54 \\
\hline & EXPERIMENTAL & & & & & & & & \\
\hline & Ambient Hummock & $-26.2 \pm 24$ & $999 \pm 805$ & $-64 \pm 4$ & $18 \pm 9$ & $30 \pm 7$ & $16 \pm 2$ & 3.2 & 0.75 \\
\hline & Warmed Hummock & $-23.0 \pm 15$ & $999 \pm 994$ & $-57 \pm 15$ & $30 \pm 11$ & $30 \pm 5$ & $17 \pm 16$ & 5.4 & 0.54 \\
\hline & Ambient Hollow & $-40.5 \pm 15$ & $999 \pm 729$ & $-58 \pm 1$ & $7 \pm 1$ & $13 \pm 0$ & $3 \pm 0$ & 3.3 & 0.82 \\
\hline & Warmed Hollow & $-26.1 \pm 18$ & $999 \pm 753$ & $-82 \pm 6$ & $30 \pm 8$ & $15 \pm 9$ & $20 \pm 6$ & 5.0 & 0.62 \\
\hline & DRAINED & & & & & & & & \\
\hline & Ambient Hummock & $-35.9 \pm 9$ & $950 \pm 440$ & $-118 \pm 8$ & $30 \pm 11$ & $12 \pm 2$ & $10 \pm 3$ & 1.0 & 0.82 \\
\hline & Warmed Hummock & $-35.6 \pm 14$ & $999 \pm 398$ & $-111 \pm 3$ & $30 \pm 6$ & $15 \pm 8$ & $20 \pm 8$ & 1.0 & 0.80 \\
\hline & Ambient Hollows & $-29.2 \pm 17$ & $850 \pm 639$ & $-71 \pm 32$ & $30 \pm 17$ & $11 \pm 3$ & $10 \pm 10$ & 0.9 & 0.68 \\
\hline & Warmed Hollow & $-19.4 \pm 27$ & $998 \pm 779$ & $-124 \pm 6$ & $21 \pm 10$ & $9 \pm 2$ & $2 \pm 2$ & 3.2 & 0.56 \\
\hline \multirow[t]{15}{*}{2012} & CONTROL & & & & & & & & \\
\hline & Ambient Hummock & $-24.8 \pm 5$ & $900 \pm 286$ & $-35 \pm 11$ & $30 \pm 14$ & $15 \pm 2$ & $10 \pm 4$ & 1.8 & 0.86 \\
\hline & Warmed Hummock & $-32.9 \pm 5$ & $999 \pm 335$ & $-54 \pm 10$ & $30 \pm 17$ & $21 \pm 1$ & $9 \pm 1$ & 1.9 & 0.85 \\
\hline & Ambient Hollow & $-21.8 \pm 4$ & $950 \pm 299$ & $-42 \pm 14$ & $30 \pm 20$ & $15 \pm 1$ & $10 \pm 2$ & 1.1 & 0.85 \\
\hline & Warmed Hollow & $-38.8 \pm 12$ & $999 \pm 382$ & $-25 \pm 43$ & $30 \pm 9$ & $17 \pm 1$ & $10 \pm 2$ & 1.2 & 0.85 \\
\hline & EXPERIMENTAL & & & & & & & & \\
\hline & Ambient Hummock & $-28.4 \pm 10$ & $998 \pm 638$ & $-29 \pm 12$ & $30 \pm 12$ & $20 \pm 4$ & $12 \pm 11$ & 2.2 & 0.75 \\
\hline & Warmed Hummock & $-27.8 \pm 26$ & $500 \pm 463$ & $-42 \pm 31$ & $30 \pm 12$ & $17 \pm 21$ & $20 \pm 5$ & 3.4 & 0.74 \\
\hline & Ambient Hollow & $-40.1 \pm 18$ & $999 \pm 574$ & $-73 \pm 3$ & $30 \pm 4$ & $11 \pm 0$ & $3 \pm 1$ & 2.4 & 0.74 \\
\hline & Warmed Hollow & $-12.6 \pm 5$ & $738 \pm 679$ & $-48 \pm 13$ & $23 \pm 11$ & $20 \pm 2$ & $5 \pm 3$ & 7.4 & 0.50 \\
\hline & DRAINED & & & & & & & & \\
\hline & Ambient Hummock & $-50.0 \pm 14$ & $950 \pm 416$ & $-105 \pm 3$ & $30 \pm 3$ & $10 \pm 2$ & $10 \pm 2$ & 0.9 & 0.82 \\
\hline & Warmed Hummock & $-50.0 \pm 19$ & $950 \pm 444$ & $-107 \pm 2$ & $30 \pm 4$ & $11 \pm 3$ & $12 \pm 1$ & 0.9 & 0.83 \\
\hline & Ambient Hollow & $-31.3 \pm 10$ & $850 \pm 246$ & $-100 \pm 11$ & $23 \pm 7$ & $10 \pm 6$ & $10 \pm 5$ & 1.6 & 0.83 \\
\hline & Warmed Hollow & $-18.5 \pm 28$ & $999 \pm 779$ & $-125 \pm 6$ & $21 \pm 13$ & $8 \pm 1$ & $1 \pm 1$ & 4.1 & 0.55 \\
\hline \multirow[t]{15}{*}{2013} & $\overline{\text { CONTROL }}$ & & & & & & & & \\
\hline & Ambient Hummock & $-32.1 \pm 5$ & $837 \pm 219$ & $-51 \pm 7$ & $30 \pm 12$ & $11 \pm 7$ & $20 \pm 4$ & 1.8 & 0.68 \\
\hline & Warmed Hummock & $-38.7 \pm 10$ & $500 \pm 136$ & $-45 \pm 6$ & $30 \pm 20$ & $20 \pm 9$ & $20 \pm 4$ & 1.1 & 0.85 \\
\hline & Ambient Hollow & $-46.8 \pm 5$ & $554 \pm 121$ & $-31 \pm 1$ & $12 \pm 1$ & $9 \pm 1$ & $6 \pm 1$ & 5.4 & 0.85 \\
\hline & Warmed Hollow & $-45.5 \pm 24$ & $500 \pm 157$ & $-24 \pm 8$ & $23 \pm 9$ & $19 \pm 5$ & $11 \pm 10$ & 1.2 & 0.85 \\
\hline & EXPERIMENTAL & & & & & & & & \\
\hline & Ambient Hummock & $-38.0 \pm 13$ & $634 \pm 292$ & $-63 \pm 3$ & $30 \pm 4$ & $14 \pm 5$ & $10 \pm 17$ & 3.1 & 0.85 \\
\hline & Warmed Hummock & $-28.3 \pm 28$ & $500 \pm 314$ & $-67 \pm 2$ & $13 \pm 2$ & $15 \pm 7$ & $20 \pm 10$ & 3.4 & 0.85 \\
\hline & Ambient Hollow & $-26.3 \pm 14$ & $500 \pm 156$ & $-64 \pm 9$ & $30 \pm 8$ & $15 \pm 7$ & $13 \pm 9$ & 2.1 & 0.85 \\
\hline & Warmed Hollow & $-16.9 \pm 14$ & $557 \pm 461$ & $-74 \pm 5$ & $11 \pm 5$ & $20 \pm 26$ & $17 \pm 12$ & 0.4 & 0.85 \\
\hline & DRAINED & & & & & & & & \\
\hline & Ambient Hummock & $-50.0 \pm 37$ & $500 \pm 180$ & $-102 \pm 3$ & $26 \pm 7$ & $30 \pm 26$ & $9 \pm 5$ & 0.6 & 0.82 \\
\hline & Warmed Hummock & $-50.0 \pm 41$ & $500 \pm 241$ & $-115 \pm 5$ & $28 \pm 7$ & $21 \pm 18$ & $9 \pm 11$ & 2.0 & 0.85 \\
\hline & Ambient Hollow & $-40.0 \pm 41$ & $820 \pm 591$ & $-76 \pm 2$ & $2 \pm 1$ & $18 \pm 5$ & $2 \pm 2$ & 1.5 & 0.83 \\
\hline & Warmed Hollow & $-28.0 \pm 43$ & $500 \pm 456$ & $-89 \pm 17$ & $7 \pm 29$ & $16 \pm 1$ & $1 \pm 0$ & 1.6 & 0.85 \\
\hline
\end{tabular}

${ }^{\mathrm{a}}$ The models were developed for each microform type $(n=3)$ at the control, experimental and drained sites separately for growing seasons of 2011, 2012 and 2013. PAR, WT and T represent photosynthetically active radiation, water table (negative values show belowground water level) and $5 \mathrm{~cm}$ soil temperature, respectively. $P_{\max }$ denotes potential maximum rate of $\mathrm{GPP}_{\mathrm{ff}}$ and $k$ is level of PAR at which half of GPP $\mathrm{Pf}_{\mathrm{ff}} \mathrm{occurs}_{\text {. WT }} \mathrm{Wpt}_{\mathrm{ot}}$ and $T_{\text {opt }}$ are Gaussian response parameters for optimum $\mathrm{GPP}_{\mathrm{ff}}$ while $\mathrm{WT}_{\mathrm{tol}}$ and $T_{\mathrm{tol}}$ are Gaussian response parameters describing the width of the curve. All modeled parameters are significant at $p=0.05$ level. 
Supplement Table S1. Estimated regression coefficient values ( \pm SE), standard error of the estimate (SEE) and goodness of fit $\left(r^{2}\right)$ for the forest floor respiration $\left(R_{\mathrm{ff}}\right)$ model (Eq. 2) ${ }^{\text {a }}$.

\begin{tabular}{|c|c|c|c|c|c|c|c|}
\hline Year & Site & $\begin{array}{l}\text { Warming Treatment / } \\
\text { Microform }\end{array}$ & $a$ & b & c & $\begin{array}{c}\text { SEE } \\
\mathrm{g} \mathrm{CO}_{2} \mathrm{~m}^{-2} \mathrm{~d}^{-1}\end{array}$ & $r^{2}$ \\
\hline \multirow[t]{12}{*}{2011} & \multirow[t]{4}{*}{ CONTROL } & Ambient Hummock & $1.30 \pm 0.3$ & $-0.21 \pm 0.1$ & $-18.3 \pm 6.8$ & 0.8 & 0.81 \\
\hline & & Warmed Hummock & $0.49 \pm 0.1$ & $-0.16 \pm 0.0$ & $-1.8 \pm 2.5$ & 3.8 & 0.37 \\
\hline & & Ambient Hollow & $1.70 \pm 0.3$ & $-0.61 \pm 0.2$ & $-32.3 \pm 10.7$ & 0.7 & 0.79 \\
\hline & & Warmed Hollow & $0.54 \pm 0.1$ & $-0.38 \pm 0.1$ & $-8.1 \pm 2.0$ & 1.8 & 0.75 \\
\hline & \multirow[t]{4}{*}{ EXPERIMENTAL } & Ambient Hummock & $1.32 \pm 0.1$ & $-0.07 \pm 0.0$ & $-5.7 \pm 2.0$ & 2.0 & 0.83 \\
\hline & & Warmed Hummock & $0.71 \pm 0.1$ & $-0.06 \pm 0.1$ & $-3.9 \pm 3.6$ & 2.8 & 0.56 \\
\hline & & Ambient Hollow & $0.67 \pm 0.2$ & $-0.37 \pm 0.1$ & $-17.1 \pm 4.7$ & 5.5 & 0.51 \\
\hline & & Warmed Hollow & $1.65 \pm 0.3$ & $-0.15 \pm 0.1$ & $-18.5 \pm 5.1$ & 5.3 & 0.60 \\
\hline & \multirow[t]{4}{*}{ DRAINED } & Ambient Hummock & $0.31 \pm 0.3$ & $-0.02 \pm 0.0$ & $2.62 \pm 7.3$ & 0.4 & 0.49 \\
\hline & & Warmed Hummock & $0.66 \pm 0.1$ & $-0.09 \pm 0.0$ & $-7.2 \pm 4.2$ & 3.4 & 0.45 \\
\hline & & Ambient Hollow & $0.55 \pm 0.1$ & $-0.02 \pm 0.0$ & $2.3 \pm 3.6$ & 0.2 & 0.85 \\
\hline & & Warmed Hollow & $1.80 \pm 0.1$ & $-0.10 \pm 0.1$ & $-20.7 \pm 4.7$ & 3.6 & 0.84 \\
\hline \multirow[t]{12}{*}{2012} & \multirow[t]{4}{*}{ CONTROL } & Ambient Hummock & $0.39 \pm 0.2$ & $-0.25 \pm 0.1$ & $-10.7 \pm 5.3$ & 0.6 & 0.53 \\
\hline & & Warmed Hummock & $0.57 \pm 0.1$ & $-0.20 \pm 0.1$ & $-1.3 \pm 2.2$ & 2.9 & 0.57 \\
\hline & & Ambient Hollow & $0.93 \pm 0.1$ & $-0.25 \pm 0.1$ & $-12.8 \pm 1.8$ & 0.4 & 0.81 \\
\hline & & Warmed Hollow & $0.90 \pm 0.1$ & $-0.16 \pm 0.1$ & $-2.8 \pm 2.0$ & 3.1 & 0.71 \\
\hline & \multirow[t]{4}{*}{ EXPERIMENTAL } & Ambient Hummock & $0.61 \pm 0.1$ & $-0.22 \pm 0.1$ & $-9.4 \pm 2.9$ & 3.2 & 0.68 \\
\hline & & Warmed Hummock & $1.08 \pm 0.1$ & $-0.15 \pm 0.1$ & $-13.5 \pm 4.9$ & 4.6 & 0.62 \\
\hline & & Ambient Hollow & $1.56 \pm 0.2$ & $-0.18 \pm 0.1$ & $-19.6 \pm 9.4$ & 6.0 & 0.67 \\
\hline & & Warmed Hollow & $0.63 \pm 0.1$ & $-0.25 \pm 0.0$ & $-12.4 \pm 1.4$ & 3.2 & 0.85 \\
\hline & \multirow[t]{4}{*}{ DRAINED } & Ambient Hummock & $0.65 \pm 0.1$ & $-0.03 \pm 0.1$ & $-3.4 \pm 8.1$ & 0.3 & 0.91 \\
\hline & & Warmed Hummock & $0.89 \pm 0.1$ & $-0.00 \pm 0.0$ & $4.4 \pm 4.5$ & 4.4 & 0.60 \\
\hline & & Ambient Hollow & $1.07 \pm 0.1$ & $-0.15 \pm 0.0$ & $-17.3 \pm 5.6$ & 0.9 & 0.80 \\
\hline & & Warmed Hollow & $1.27 \pm 0.1$ & $-0.00 \pm 0.0$ & $-1.5 \pm 3.7$ & 5.0 & 0.67 \\
\hline \multirow[t]{12}{*}{2013} & \multirow[t]{4}{*}{ CONTROL } & Ambient Hummock & $1.74 \pm 0.1$ & $-0.01 \pm 0.1$ & $-5.3 \pm 3.1$ & 3.0 & 0.67 \\
\hline & & Warmed Hummock & $2.02 \pm 0.2$ & $-0.03 \pm 0.0$ & $-8.3 \pm 3.4$ & 4.2 & 0.56 \\
\hline & & Ambient Hollow & $1.18 \pm 0.1$ & $-0.15 \pm 0.1$ & $-3.8 \pm 1.7$ & 3.1 & 0.68 \\
\hline & & Warmed Hollow & $1.56 \pm 0.2$ & $-0.18 \pm 0.1$ & $-5.4 \pm 2.1$ & 3.7 & 0.71 \\
\hline & \multirow[t]{4}{*}{ EXPERIMENTAL } & Ambient Hummock & $0.75 \pm 0.5$ & $-0.53 \pm 0.1$ & $-10.3 \pm 3.3$ & 3.5 & 0.70 \\
\hline & & Warmed Hummock & $1.29 \pm 0.3$ & $-0.47 \pm 0.1$ & $-45.9 \pm 7.5$ & 6.2 & 0.53 \\
\hline & & Ambient Hollow & $0.48 \pm 0.3$ & $-0.61 \pm 0.1$ & $-29.8 \pm 2.5$ & 2.5 & 0.83 \\
\hline & & Warmed Hollow & $0.96 \pm 0.3$ & $-0.19 \pm 0.1$ & $-10.3 \pm 5.5$ & 6.9 & 0.38 \\
\hline & \multirow{4}{*}{ DRAINED } & Ambient Hummock & $1.44 \pm 0.2$ & $-0.17 \pm 0.0$ & $-27.5 \pm 2.7$ & 2.8 & 0.82 \\
\hline & & Warmed Hummock & $2.17 \pm 0.2$ & $-0.17 \pm 0.0$ & $-32.1 \pm 3.2$ & 2.9 & 0.80 \\
\hline & & Ambient Hollow & $1.7 \pm 0.3$ & $-0.32 \pm 0.0$ & $-41.5 \pm 5.5$ & 6.5 & 0.61 \\
\hline & & Warmed Hollow & $0.77 \pm 0.2$ & $-0.20 \pm 0.0$ & $23.2 \pm 4.8$ & 3.5 & 0.63 \\
\hline
\end{tabular}

${ }^{\mathrm{a}}$ The models were developed for each microform type $(n=3)$ at the control, experimental and drained sites separately for growing seasons of 2011, 2012 and 2013. $a, b$ and $c$ are regression coefficients. Negative values of b represent greater respiration with deeper water table values (below-ground WT having negative values). All modeled parameters are significant at $p=0.05$ level. 